\title{
Perfil de la inserción externa y conducta exportadora de PYMES del Sector químico de Venezuela
}

\author{
Pardo Bernardoni, Matias \\ Broward College, Miami, Estado de Florida, Estados Unidos de \\ Norteamérica \\ matipardoni@gmail.com
}

Recibido: 28 febrero 2018 Aprobado: 20 marzo 2018

\begin{abstract}
Resumen
La presente investigación tiene como propósito la aplicación del Modelo Moori-Koenig-Yoguel para determinar el perfil de la inserción externa y conducta exportadora a Pymes de del sector químico de Venezuela, la cual se basa en la fundamentación teórica de Moori-Koenig-Yoguel (1995), Carvajal (2014), para la variable inserción externa y por Estrada y Heijs (2005) y Gatto (1995) para conducta exportadora, la misma tuvo un enfoque metodológico positivista, fue de metodología empleada fue de tipo analítica, con un diseño, no experimental transeccional-descriptiva de campo. La población estuvo conformada por tres empresas seleccionadas al azar con tres unidades de información correspondiendo a Gerentes de las tres empresas, debido a que se trabajó bajo un muestreo de tipo incidental. Para la ejecución de la misma se diseñó un instrumento identificado como "EXPORTES" contentivo de ambas variables el cual fue sometido a juicio de experto por tres especialistas y luego se obtuvo la confiabilidad de este bajo el método Alpha de Cronbach, arrojando una confiabilidad 0.90. Los datos obtenidos fueron interpretados a través de la aplicación de estadísticas no paramétricas de indicadores medidos, mostrando la existencia de debilidades a nivel de la dinámica exportadora, tipología y perfil básico para la variable inserción externa. Por parte de la variable conducta exportadora las debilidades se encuentran a nivel de los indicadores inserción externa y proactividad exportadora. Partiendo de esta información, se pasó a diseñar una propuesta teórica con lineamientos para hacerle frente a la problemática evidenciada en la investigación.
\end{abstract}

Palabras clave: Inserción externa; conducta exportadora; PYMES. 


\title{
Profile of the external insertion and export behavior of PYMES of the Chemical Sector of Venezuela
}

\begin{abstract}
The present investigation has as purpose the application of the Moori-Koenig-Yoguel Model to determine the profile of the external insertion and export behavior to SMEs of the chemical sector of Venezuela., The same, its theoretical foundation by Moori-Koenig-Yoguel (1995), Carvajal (2014), for the variable external insertion and by Estrada and Heijs (2005) and Gatto (1995) for export behavior, it had a positivist methodological approach, it was the methodology used was analytical, with a design, non-experimental transectional-descriptive field. The population consisted of three companies selected at random with three units of information corresponding to managers of the three companies, because they worked under an incidental sampling. For the execution of the same instrument was designed identified as "EXPORTS" containing both variables which was submitted to expert judgment by three specialists and then the reliability was obtained under the Alpha method of Cronbach, yielding a reliability of 0.90. The data obtained were interpreted through the application of nonparametric statistics of measured indicators, showing the existence of weaknesses at the level of export dynamics, typology and basic profile for the external insertion variable. On the part of the export behavior variable, weaknesses are found at the level of external insertion indicators and export proactivity. Based on this information, we went on to design a theoretical proposal with guidelines to deal with the problems highlighted in the research.
\end{abstract}

Keywords: External insertion; export behavior; PYMES.

\section{Introducción}

En el contexto mundial las cooperativas

$\mathrm{y}$ pequeñas y medianas empresas o llamadas también microempresa ha venido ocupando la mayoría del número de empresas por tamaño y su participación en la generación de empleo igualmente es mayoritaria, esto tiene que ver con un tema de asociatividad como lo indica Coba, Díaz y Sánchez (2016); sin embargo, su estructura y gestión son vulnerables, debido al grado de aptitud para la administración de sus negocios, la escasa capacitación, participación en mercados externos así como problemas de liquidez en sus activos, suelen ser los mayores problemas de sustentabilidad que enfrentan este sector. Lo anteriormente se sustenta en lo referido por autores como Bucardo (2007), en su investigación realizada para el Banco Interamericano de Desarrollo (BID), quien señaló que la microempresa padece de ciertas debilidades, como alta informalidad en el sector de Autoempleo, baja capacidad empresarial, baja capacidad tecnológica y bajo nivel educativo. En el mismo informe, se menciona que el segmento presenta problemas financieros, tales como problemas de cobranzas, falta de capital de trabajo, capital propio y recursos de crédito.

De acuerdo con Lora(2013), las empresas pequeñas y medianas son más numerosas que las grandes en todos los países, pero en América Latina, tiene una sobreabundancia de empresas extremadamente pequeñas. En Estados Unidos, 
por ejemplo, el 54\% de las empresas tiene 10 trabajadores o menos; en Argentina, esa cifra es del 84\%; y, en México y Bolivia supera el 90\%. Este mismo autor acota que esta situación se replica en todos los países de Latinoamérica, incluyendo a economías como las de Chile y Brasil.

La conducta exportadora en este momento el nivel de exportación de acuerdo al informe del (BID, 2016), el nivel de ventas externas de acuerdo en: Suramérica: $-46 \%$, América Latina y el Caribe: $-57 \%$, EEUU: $-28 \%$, Asia (excluye China): $-58 \%$, China: $-31 \%$,Unión Europea: $-36 \%$, Total Mundo: $-49 \%$. En el caso de Venezuela, el referido informe del BID señala que hizo sus estimaciones sobre cifras no oficiales, tomando los datos de la Organización de Países Exportadores de Petróleo y del Fondo Monetario Internacional, medido en dólares estadounidenses, las ventas totales externas de Venezuela alcanzaron los 38 mil millones de dólares, con una caída del 36\% respecto al año 2014.

En el caso de Venezuela, se puede presentar un grupo de pequeñas y medianas empresas (PYMES), las cuales se tornan interesantes poder determinar las dimensiones de datos básicos, indicadores de perfomance, tipología exportadora y actitud proactiva o pasiva en la búsqueda de clientes externos, ya que se puede inferir de observación informal que guardan similitud de haber surgido como emprendimientos a pequeña escala local y hoy muestran conductas exportadoras.

Para ello se adoptó el Modelo MooriKoenig \& Youguel, CEPAL (1995), es una propuesta de metodología de análisis la cual fue aplicada por primera vez por la CEPAL en un estudio en PYMES para la Comisión Económica para América Latina y el Caribe en Argentina, el cual consta de dos variables y quince factores que permiten medir cuatro dimensiones determinantes del perfil de inserción y conducta exportadora. Para efectos de este estudio se asume este modelo teórico el cual se lo ha nombrado como el "Gran Menú" de este axioma para el desarrollo operacional de un cuestionario original que permita lograr la resolución del problema planteado y que a continuación se sistematiza.

A la luz de lo investigado por Hurtado (2016), se entenderá por inserción interna al Coeficiente de Apertura Externa (CAE) o Grado de Apertura Externa mide el peso del comercio 
exterior de un país respecto al producto interior. Con ello se pretende conocer en qué medida una empresa puede estar llevando a cabo un proceso de internacionalización de su economía a lo largo de los años, o si por el contrario está cerrando sus fronteras, a través de un crecimiento de las barreras proteccionistas. Por su parte la variable se entiende como las exportaciones de un país reflejan la eficiencia productiva de las empresas de forma ex post, es decir, se trata de evaluar la competitividad por medio de sus efectos reales en el mercado (Estrada y Heijs 2005)

Tomando en cuenta lo anteriormente referido, se formula la siguiente interrogante ¿Será posible la aplicación del modelo MooriKoenig- Youguel para determinar el perfil de la inserción externa y conducta exportadora en Pymes del sector Químico de Venezuela? Para dar respuesta a esta interrogante se planteó como objetivo general aplicar el modelo MooriKoenig- Youguel para determinar el perfil de la inserción externa y conducta exportadora en Pymes del sector Químico de Venezuela.

\section{Materiales y método}

La metodología como herramienta esencial para desarrollar la investigación permitió relacionar los objetivos planteados con los datos arrojados de la realidad, aplicar el instrumento. El tipo de investigación se orientó a ser descriptiva, aplicada, de diseño transversal, descriptivo de campo, no experimental.

La población estuvo integrada por las empresas PYMES del sector químico de la ciudad de Maracaibo, estado Zulia, Venezuela. Como muestra se seleccionaron al azar las tres primeras empresas en del ranking, las cuales por razones de confidencialidad de la información serán identificadas como $\mathrm{A}, \mathrm{B}, \mathrm{C}$, asumiendo de ellas dos unidades de información (Gerente General y Gerente de Comercialización), siendo la muestra total de seis unidades de información.

Partiendo de las preconizaciones de los investigadores Moori- Koenig-Yoguel (1995), desarrollaron un constructo teórico analítico el cual llamarón el gran menú, el cual consiste en una matriz conformada por componentes, factores y elementos los cuales en forma decreciente permiten evaluar las variables referidas a inserción externa y conducta exportadora. El primer componente Inserción externa está compuesto por dos factores y nueve elementos. Por su parte, el componente Conducta exportadora comprende de dos factores y seis elementos. De acuerdo a los autores antes referidos el modelo solo sirve como 
referente teórico del cual se puede diseñar los cuestionarios que permitan escrutar cada uno de los elementos que definirán a su vez los factores y definir el comportamiento de los componentes del gran menú propuesto y que operacionalizan el presente estudio, Grafica 01.

De acuerdo a estos basamentos teóricos y a la metodología seguida por Carrasquero (2012), se confeccionaron dos instrumentos de recolección de datos fue diseño de los investigadores fue mediante un cuestionario, integrado por 50 ítems, 25 para cada variable en estudio para efectos de la aplicación. El cuestionario está estructurado mediante dos secciones, la primera que integra el perfil de datos básicos, y la segunda que da respuesta a a cada una de las dimensiones e indicadores.

\begin{tabular}{|c|c|c|c|c|c|}
\hline Componente & \multicolumn{3}{|c|}{ Inserción Externa } & \multicolumn{2}{|c|}{ Conducta Exportadora } \\
\hline Factor & \multicolumn{3}{|c|}{$\begin{array}{c}\text { Perfil de datos básicos e indicadores claves } \\
\text { performance }\end{array}$} & Tipología exportadora & $\begin{array}{l}\text { Actitud Proactiva o pasiva en } \\
\text { La búsqueda de clientes }\end{array}$ \\
\hline \multirow[t]{6}{*}{ Elementos } & \multirow[t]{2}{*}{$\begin{array}{l}\text { Oferta } \\
\text { exportadora }\end{array}$} & \multirow[t]{2}{*}{$\begin{array}{l}\text { Dinámica de } \\
\text { exportación }\end{array}$} & \multirow[t]{2}{*}{$\begin{array}{l}\text { Tamaño de } \\
\text { la PyMES }\end{array}$} & \multirow[t]{2}{*}{$\begin{array}{l}\text { Excelencia } \\
\text { exportadora }\end{array}$} & Imaginación \\
\hline & & & & & Creatividad \\
\hline & \multirow{2}{*}{$\begin{array}{l}\text { Tamaño y } \\
\text { grado de } \\
\text { inserción }\end{array}$} & \multirow{2}{*}{$\begin{array}{l}\text { Grado de } \\
\text { afectación }\end{array}$} & \multirow{2}{*}{$\begin{array}{l}\text { Mercado de } \\
\text { destino }\end{array}$} & \multirow{2}{*}{$\begin{array}{l}\text { Sendero de } \\
\text { excelencia }\end{array}$} & \\
\hline & & & & & Construccion \\
\hline & \multirow{2}{*}{$\begin{array}{c}\text { Ambigüedad } \\
\text { de } \\
\text { exportación }\end{array}$} & Proceso de & Localización & Inserción externa & \\
\hline & & & & Oportunidad & \\
\hline
\end{tabular}

Figura 1. Gran Menú de Modelo Moori- Koenig-Yoguel (1995)

Fuente: Pardo, (2018)

Los instrumentos ofrecen cinco (5) de conducta exportadora 0.90 .

opciones de respuesta: Siempre, Casi Siempre, A veces, Casi Nunca y Nunca; las cuales reciben un puntaje en el rango de 1 y 5 puntos y respuestas dicotómicas. Se aplicaron criterios de validez a través de la técnica de expertos. La confiabilidad, fue determinada a través que el instrumento de inserción externa obtuvo un coeficiente de Alfa de Cronbrach de 0,91, mientras que el instrumento

En el caso que nos ocupa, los datos fueron tratados a través del programa SPSS versión 23, siguiendo el siguiente procedimiento:

a) Construcción de 50 variables, donde fueron definidas las etiquetas y características de cada una de ellas (Variable, tipo, achura, decimales, etiqueta, valores, perdidos, 
columnas alineación, medida y rol);

b) Carga de los datos en la plantilla de la vista de datos;

c) Para el análisis se siguió la técnica de agrupación por dimensiones y variable.

d) Creadas las subvariables agrupadas por dimensiones y variables se les analizo a través de la técnica de agrupación visual.

e) Las agrupaciones visuales fueron analizadas siguiendo a través de los estadísticos descriptivos: frecuencia, Variación, media, desviación estándar, suma.

\section{Análisis y discusión de resultados}

A continuación, se analizan los resultados enfocados en aplicación del Modelo MooriKoenig- Youguel para determinar el perfil de la inserción externa y conducta exportadora de Pymes del sector químico de Venezuela.

Como protocolo de presentación los resultados encontrados estos se muestran siguiendo el orden de presentación de los objetivos que fueron planteados en el cuadro operacional que sirvió de guiar para la investigación, junto a sus respectivas dimensiones e indicadores. Finalmente se establecerán las generalizaciones que permitan a través de la discusión reflexiva, contrastar los resultados de acuerdo a los basamentos teóricos y las investigaciones que sirvieron de antecedentes a la misma.

\section{Análisis de la variable inserción externa}

La aplicación del instrumento diseñado para la determinación de la inserción externa, aplicado a la muestra, permitió reportar los siguientes resultados (Cuadro 1).

De los resultados tabulados se puede destacar que la oferta exportadora de las empresas estudiadas es $66.77 \%$ ("Muy buena") y un 33,33\% ("Buena), de donde se infiere que los resultados permitieron medir que la tendencia es hacia la especialización de los productos exportados por la empresa, los cuales poseen una buena condición técnica dentro de los mercados donde exporta, y cumple con los estándares internacionales, como son las Normas Cofrac ESSAIS y IQ NET. Por otra parte, se observó que los elementos de valor de los productos exportados por las empresas en estudio dentro de las exigencias técnicas de los productos y el nivel de especialización de la oferta frente a los productos locales donde tienen presencia. En el espectro de la muestra se perciben que algunas de ellas siguen teniendo falencias en cuando a 
las exigencias técnicas posiblemente por poco desarrollo de especificidades técnicas.

Los resultados encontrados referentes a indicador tamaño y grado de inserción, los niveles de facturación se encuentran en los rangos de BsF: 10.000 .000 y 109.000.000. Con una fuerza laboral entre 75 a 100 empleados. En cuanto a los niveles de exportación en dólares se encuentran dentro de los rangos de $\$ .3 \mathrm{~K}$ y los $\$$. $10 \mathrm{~K}$.

\section{Tabla 1}

Ambigüedad Exportadora
La ambigüedad de exportación, entendida esta como el tiempo transcurrido desde la creación de la empresa hasta el inicio de exportaciones se observan que inician en el periodos 2007 al 2017, lo que se traduce en un índice de ambigüedad de 15, 20 y 28 años. Este comportamiento en todos los casos se ve activados por la necesidad de conseguir divisas para poder compensar los gastos operativos y la compra de la materia prima en el extranjero por falta de políticas públicas claras.

\begin{tabular}{|c|c|c|c|}
\hline Empresa & $\begin{array}{c}\text { Año de } \\
\text { Fundación }\end{array}$ & $\begin{array}{c}\text { Iniciación } \\
\text { Exportaciones }\end{array}$ & $\begin{array}{l}\text { Ambigüedad } \\
\text { Exportadora }\end{array}$ \\
\hline $\mathrm{A}$ & 1992 & 2007 & 15 \\
\hline B & 1985 & 2013 & 28 \\
\hline $\mathrm{C}$ & 1996 & 2016 & 20 \\
\hline
\end{tabular}

Fuente: Pardo, (2018)

En lo referente a la Dinámica de ningún proceso de integración comercial regional exportación se observa que el $66.7 \%$ ("Regular) ni se les ha ofrecido financiamientos externos con mientras que 33.3\% ("Mala") de la muestra, otros competidores del mercado en lo interno. de acuerdo con Álvarez (2006), definió como En lo exógeno las normativas y regulaciones de las dificultades exógenas y endógenas que los exportación dificultan los procesos de inserción productos exportados tienen en el mercado exportadora de los productos en los países interno a partir de la apertura de la economía, el destino.

proceso de integración y la desregulación de los mercados. Los resultados permiten inferir que es claro que las empresas no están asociadas a

Por su parte en el caso de la Performance exportadora el $66.7 \%$ ("Siempre”) y $33.33 \%$ (“Algunas veces”). Este indicador evaluó, 
apegado a lo definido por la Moori y Yoguel(1995) la heterogeneidad de la performance exportadora de la empresa y la capacidad explicativa de distintas variables sobre ese fenómeno. Los resultados permiten observar que para las empresas en su mayoría han tenido que pasar por un proceso de aprendizaje organizacional, donde aún se requiere que se fomente la creatividad tomando casi siempre en consideración los niveles de calidad de los mercados de destino que son productos de una planificación previa de exportación pero existiendo todavía sesgos de necesidad de que requieren los mercados de destino.

En referencia al grado de afectación un $66.7 \%$ (Bueno) y un $33.3 \%$ (Siempre), observando que el grado de afectación está en función a las dificultades que se traducen por la caída del nivel de actividad racionalización de la planta para reducir costos, perdida del liderazgo en el segmento del mercado en el cual compite de acuerdo a los investigadores Moori-Koenig \& Yoguel (1995). Por otra parte particularizando en el análisis de los resultados con las empresas objeto de estudio se puede inferir que tener una buena estructura organizacional es fundamental donde se valora la conducta del colectivo, y valora regularmente el desempeño de toda la estructura organizacional, pero no existiendo una definición clara de los del impacto de la exportación de los productos. En cuanto al indicador interés por los productos en el mercado las empresas en estudio indican que la presencia de líneas muy definidas y de poca variedad.

Finalizando con el indicador Exigencias de los mercados de destino el 100\% ("Muy Bueno"), donde se valoró la percepción de la empresa sobre las exigencias normativas del mercado de destino, donde se percibe que el comportamiento del mercado en el exterior ha sido ("Muy bueno"), con igual valoración reditual del capital de trabajo y se hace hincapié por las limitaciones que se tienen el país de origen de la exportación en este caso Venezuela. 
Tabla 2

Variable Inserción Externa-Análisis de indicadores

\begin{tabular}{|c|c|c|c|c|c|c|c|c|c|}
\hline \multirow[t]{2}{*}{ Indicador } & \multicolumn{5}{|c|}{ Distribución de respuestas (\%) } & \multicolumn{4}{|c|}{ Estadísticas } \\
\hline & $\begin{array}{c}\text { Muy } \\
\text { buena } \\
\text { Siem } \\
\text { pre } \\
\text { Ponde- } \\
\text { ración } \\
\text { (5) }\end{array}$ & $\begin{array}{c}\text { Buena } \\
\text { Casi } \\
\text { Siem- } \\
\text { pre } \\
\text { Ponde- } \\
\text { ración } \\
\text { (4) }\end{array}$ & $\begin{array}{c}\text { Regular } \\
\text { Algu- } \\
\text { nas ve- } \\
\text { ces } \\
\text { Ponde- } \\
\text { ración } \\
\text { (3) }\end{array}$ & $\begin{array}{c}\text { Mala } \\
\text { Casi } \\
\text { Nunca } \\
\text { Ponde- } \\
\text { ración } \\
(2)\end{array}$ & $\begin{array}{c}\text { Muy } \\
\text { Mala } \\
\text { Nunca } \\
\text { Ponde- } \\
\text { ración } \\
\text { (1) }\end{array}$ & Sun & Var & $\begin{array}{r}\text { Desv } \\
\text { Est }\end{array}$ & Med \\
\hline $\begin{array}{l}\text { Oferta } \\
\text { exportadora }\end{array}$ & 66,7 & 33.33 & 0.0 & 0.0 & 0.0 & 100 & 33.3 & 0.58 & 5.0 \\
\hline $\begin{array}{l}\text { Dinámica de } \\
\text { exportación }\end{array}$ & 0.0 & 0.0 & 66.7 & 33.33 & 0.0 & 100 & 0.12 & 0.35 & 1.80 \\
\hline $\begin{array}{l}\text { Performance } \\
\text { exportadora }\end{array}$ & 66.7 & 0.0 & 33.3 & 0.0 & 0.0 & 100 & 0.70 & 0.86 & 4.50 \\
\hline $\begin{array}{l}\text { Grado de } \\
\text { afectación }\end{array}$ & 33.3 & 66.7 & 0.0 & 0.0 & 0.0 & 100 & 0.33 & 0.58 & 4.30 \\
\hline $\begin{array}{l}\text { Interés por los } \\
\text { productos }\end{array}$ & 0.0 & 66.7 & 33.7 & 0.0 & 0.0 & 100 & 0.70 & 0.86 & 4.50 \\
\hline $\begin{array}{l}\text { Exigencias de } \\
\text { los mercados } \\
\text { destinos }\end{array}$ & 100.0 & 0.0 & 0.0 & 0.0 & 0.0 & 100 & 0.00 & 0.00 & 5.0 \\
\hline
\end{tabular}

Fuente: Pardo, (2018)

Entérminosgenerales delcomportamiento las exigencias de los mercados endógenos y de la variable inserción externa es de $4.78 \%$ exógenos, lo que podría estar relacionado con la ("Buena"), la cual se obtuvo del promedio de antigüedad de exportación, así como a nivel de la la sumatoria de todos los indicadores, donde se dimensión tipología exportadora.

pudo conocer en qué medida las empresas objeto de estudio puede estar llevando a cabo un proceso de internacionalización de su economía a lo largo de los años, (Hurtado Cañas, 2016). Se pueden identificar que a través del análisis general que existen falencias y barreras en torno a indicadores como la performance, el grado de afectación y 
Tabla 3

Variable Inserción Externa-Análisis de Dimensiones

\begin{tabular}{|c|c|c|c|c|c|c|c|c|c|}
\hline \multirow[t]{2}{*}{ Dimensión } & \multicolumn{5}{|c|}{ Distribución de respuestas (\%) } & \multicolumn{4}{|c|}{ Estadísticas } \\
\hline & $\begin{array}{c}\text { Muy } \\
\text { buena } \\
\text { Siem- } \\
\text { pre } \\
\text { Ponde- } \\
\text { ración } \\
\text { (5) } \\
\end{array}$ & $\begin{array}{c}\text { Buena } \\
\text { Casi } \\
\text { Siem- } \\
\text { pre } \\
\text { Ponde- } \\
\text { ración } \\
(4) \\
\end{array}$ & $\begin{array}{c}\text { Regular } \\
\text { Algu- } \\
\text { nas ve- } \\
\text { ces } \\
\text { Ponde- } \\
\text { ración } \\
\text { (3) }\end{array}$ & $\begin{array}{c}\text { Mala } \\
\text { Casi } \\
\text { Nunca } \\
\text { Ponde- } \\
\text { ración } \\
(2)\end{array}$ & $\begin{array}{c}\text { Muy } \\
\text { Mala } \\
\text { Nunca } \\
\text { Ponde- } \\
\text { ración } \\
\text { (1) }\end{array}$ & Sun & Var & $\begin{array}{c}\text { Desv } \\
\text { Est }\end{array}$ & Med \\
\hline Perfil básico & 100.0 & 0.0 & 0.0 & 0.0 & 0.0 & 100 & 0.0 & 0.0 & 5.0 \\
\hline $\begin{array}{l}\text { Indicadores Cla- } \\
\text { ves de la Perfor- } \\
\text { mance }\end{array}$ & 100.0 & 0.0 & 0.0 & 0.0 & 0.0 & 100 & 0.0 & 0.0 & 5.0 \\
\hline $\begin{array}{l}\text { Tipología Expor- } \\
\text { tadora }\end{array}$ & 66.7 & 33.3 & 0.0 & 0.0 & 0.0 & 100 & 0.33 & 0.58 & 4.33 \\
\hline
\end{tabular}

Fuente: Pardo, (2018)

\section{Análisis de la variable conducta exportadora}

Según los resultados del cuestionario diseñado para el escrutinio de la variable conducta exportadora entendida como las exportaciones de un país que reflejan la eficiencia productiva de las empresas de forma ex post, es decir, se trata de evaluar la competitividad por medio de sus efectos reales en el mercado Estrada y Heijs (2005).

Los resultados agrupados en siete indicadores en función de los análisis realizados, se puede observar que la Excelencia exportadora es en $66.7 \%$ ("Muy Alta”) y un 33.33\% (“Alta), demostrando que el mercado de destino ha ofrecido similares condiciones de financiamiento que sus competidores locales, pero con un alto esfuerzo por adaptarlos productos al mercado externo y con un muy alto grado de complejidad en las estrategias de exportación en especial cuando son continentes diferentes donde se ha logrado la inserción exportadora. En referencia a los factores endógenos que sustentan su posicionamiento competitivo la muestra refiere que están en la aplicación de tecnologías, Actualización de los procesos de acuerdo a las normas de calidad de los mercados de destino, y el aumento de las exigencias sobre la calidad de las materias primas.

El hecho de haber asumido un 
Sendero de excelencia 100\% ("Muy Alto"), exterior, frecuencia de la presencia del producto, la cual se evidencia en la posición de haber diversidad de tipos de producto y predominio del asumido Normativas internas tales como producto en mercados internos, los resultados FONDONORMA ISO, Normas Cofrac ESSAIS permiten observar que la ponderación de acuerdo y IQ NET Magnagement System, entre otras, a la media es de 4.67 (“Alta"), debido a la poca administradas por una Oficina de Control de la diversidad de tipos de productos y el nivel como Calidad y respaldado por una antigüedad de los productos en algunos casos de más de 25 años.

La inserción externa se refiere a un conjunto de características que permiten la caracterización de las Pymes de acuerdo al predominio de la firma, nivel del coeficiente de exportación, contenido importado en función de la producción, adaptadores pasivos en el mercado externo, oportunidades comerciales y nivel tecnológico (CEPAL, 1995). Para los directivos de la empresa A el nivel es de 6/10 (“Alta”), B 6/10 (“Alta") y C., 3/10 (“Baja”), sobre el nivel de predominio de la marca, por los valores de poseer un nivel tecnológico con pocos competidores en los mercados externos donde hace presencia externa.

Continuando con el indicador oportunidad entendida como las características exportadoras que permiten el nivel de inserción externa, definido por Moori-Koenig \& Yoguel (1995) como el predominio del producto en el comercio percibido en el mercado externo del predominio del producto dentro de los mercados internos.

Para efectos de esta investigación, se asumió como imaginación lo dicho por Aladi (2005), el nivel de desarrollos originales que pueda tener el producto para lograr su inserción externa, el cual de acuerdo a los resultados se reporta como 100\% ("Muy Alta"), las exigencias de técnicas gerenciales en planificación y diseño de los productos de exportación. Continuando la con el indicador Creatividad en el 100\% de los casos existe de un departamento de creatividad y de desarrollo tecnológico para la exportación. De acuerdo a Bacos y Brynjolfsson (1997) y en tanto la Construcción es definida por Camagni y Capello (2005), como la existencia de una unidad de planificación estratégica dentro de la empresa la cual si está presente

Finalmente, según Estrada y Heijs (2005), los resultados reportados para la variable Conducta exportadora es en promedio 
ponderado de la media 4,5 (“Alta"), siendo la investigación, reflejan la eficiencia productiva tipología exportadora y una actitud proactiva en de las empresas de forma ex post, es decir, tiene la búsqueda de clientes externos. Esto permite una actitud de competitividad por medio de sus inferir de acuerdo a los resultados que la conducta efectos reales en el mercado externo. exportadora de las empresas objeto de esta

\section{Tabla 4}

Variable Conducta Exportadora

\begin{tabular}{|c|c|c|c|c|c|c|c|c|c|}
\hline \multirow[t]{2}{*}{ Indicador } & \multicolumn{5}{|c|}{ Distribución de respuestas (\%) } & \multicolumn{3}{|c|}{ Estadísticas } & \multirow[b]{2}{*}{ Med } \\
\hline & $\begin{array}{c}\text { Muy } \\
\text { alto } \\
\text { Siem- } \\
\text { pre } \\
\text { Ponde- } \\
\text { ración }\end{array}$ & $\begin{array}{c}\text { Alto } \\
\text { Casi } \\
\text { Siem- } \\
\text { pre } \\
\text { Ponde- } \\
\text { ración } \\
\text { (4) }\end{array}$ & $\begin{array}{l}\text { Regular } \\
\text { Algu- } \\
\text { nas ve- } \\
\text { ces } \\
\text { Ponde- } \\
\text { ración } \\
\text { (3) }\end{array}$ & $\begin{array}{c}\text { Mala } \\
\text { Bajo } \\
\text { Casi } \\
\text { Nunca } \\
\text { Ponde- } \\
\text { ración } \\
\text { (2) }\end{array}$ & $\begin{array}{c}\text { Muy } \\
\text { Bajo } \\
\text { Nunca } \\
\text { Ponde- } \\
\text { ración } \\
\\
\text { (1) }\end{array}$ & Sun & Var & $\begin{array}{l}\text { Desv } \\
\text { Est }\end{array}$ & \\
\hline \multicolumn{10}{|l|}{$\begin{array}{l}\text { Excelencia } \\
\text { exportadora }\end{array}$} \\
\hline & 66.7 & 33.3 & 0.0 & 0.0 & 0.0 & 100 & 0.33 & 5.77 & 4.67 \\
\hline $\begin{array}{l}\text { Sendero de } \\
\text { excelencia }\end{array}$ & 100.0 & 0.0 & 0.0 & 0.0 & 0.0 & 100 & 0.0 & 0.0 & 5.0 \\
\hline $\begin{array}{l}\text { Inserción ex- } \\
\text { terna }\end{array}$ & 0.0 & 66.7 & 33.3 & 0.0 & 0.0 & 100 & 0.33 & 0.58 & 3.67 \\
\hline Oportunidad & 66.7 & 33.3 & 0.0 & 0.0 & 0.0 & 100 & 0.33 & 0.58 & 4.67 \\
\hline Imaginación & 100.0 & 0.0 & 0.0 & 0.0 & 0.0 & 100 & 0.0 & 0.0 & 5.0 \\
\hline Creatividad & 100.0 & 0.0 & 0.0 & 0.0 & 0.0 & 100 & 0.0 & 0.0 & 5.0 \\
\hline Construcción & 100.0 & 0.0 & 0.0 & 0.0 & 0.0 & 100 & 0.0 & 0.0 & 5.0 \\
\hline
\end{tabular}

Fuente: Pardo, (2018) 
Tabla 5

Variable Conducta Exportadora-Análisis de Dimensiones

\begin{tabular}{|c|c|c|c|c|c|c|c|c|c|}
\hline \multirow[t]{2}{*}{ Dimensión } & \multicolumn{5}{|c|}{ Distribución de respuestas (\%) } & \multicolumn{4}{|c|}{ Estadísticas } \\
\hline & $\begin{array}{c}\text { Muy } \\
\text { buena } \\
\text { Siem- } \\
\text { pre } \\
\text { Ponde- } \\
\text { ración } \\
(5) \\
\end{array}$ & $\begin{array}{c}\text { Buena } \\
\text { Casi } \\
\text { Siem- } \\
\text { pre } \\
\text { Ponde- } \\
\text { ración } \\
\text { (4) }\end{array}$ & $\begin{array}{c}\text { Regular } \\
\text { Algu- } \\
\text { nas ve- } \\
\text { ces } \\
\text { Ponde- } \\
\text { ración } \\
\text { (3) }\end{array}$ & $\begin{array}{c}\text { Mala } \\
\text { Casi } \\
\text { Nunca } \\
\text { Ponde- } \\
\text { ración } \\
\text { (2) }\end{array}$ & $\begin{array}{c}\text { Muy } \\
\text { Mala } \\
\text { Nunca } \\
\text { Ponde- } \\
\text { ración } \\
\text { (1) }\end{array}$ & Sun & Var & $\begin{array}{c}\text { Desv } \\
\text { Est }\end{array}$ & Med \\
\hline $\begin{array}{l}\text { Tipología Expor- } \\
\text { tadora } \\
\text { Actitud proac- } \\
\text { tiva o pasiva en } \\
\text { la búsqueda de } \\
\text { clientes }\end{array}$ & 100.0 & 100.0 & 0.0 & 0.0 & 0.0 & 100 & 0.0 & 0.0 & 5.0 \\
\hline
\end{tabular}

Fuente: Pardo, (2018)

\section{Discusión de los resultados}

Para la interpretar los resultados del presente trabajo es necesario conocer los conceptos de inserción externa y conducta exportadora. Para efectos de esta investigación se entenderá por inserción externa, al Coeficiente de Apertura Externa (CAE) o Grado de Apertura Externa mide el peso del comercio exterior de un país respecto al producto interior. Con ello se pretende conocer en qué medida una empresa puede estar llevando a cabo un proceso de internacionalización de su economía a lo largo de los años, o si por el contrario está cerrando sus fronteras, a través de un crecimiento de las barreras proteccionistas. (Hurtado Cañas, 2016).
En esta investigación la inserción externa responde más a una decisión individual de las PYMES estudiadas, ya queenningún caso obedece a una política pública de estado que incentive los procesos de exportación de los productos, bien con incentivos o apoyo financiero. De hecho, los procesos de internacionalización responden más a una coyuntura de recapitalización externa, frente a la situación de controles fiscales, económicos y de desindustrialización que existentes en Venezuela.

Profundizando aún más en los resultados reportados lo anterior se ve evidenciado en el índice de ambigüedad de exportación el cual se sitúa por encima de los 10 años, datos que son 
coincidentes con los reportados por Moori Koenig et al (1994), cuando afirma que la antigüedad exportadora de las Pymes argentinas es similar a la que surge de otras evidencias empíricas de países desarrollados que han transitado procesos de desindustrialización Samuels et al (1992), y es significativamente distinta a la observada en algunos países del sudeste asiático. Asimismo, el índice es coincidente con los hallazgos de esta investigación cuando los mismos autores reportan un índice de ambigüedad de más de 10 años de antigüedad.

Por otra parte, la tendencia hacia una baja dinámica de exportación reportada en este estudio responde a que las PYMES estudiadas enfrentan múltiples dificultades en la dinámica de los procesos de exportación, tales como: a) No contar con facilidades para la financiación de las exportaciones, b) Falta de estudios de Mercadeo confiables en los mercados objetivos que permitan una expansión confiables, c) Problemas en el despacho y los procesos de aranceles y procesos aduaneros en lo interno y en lo externo. Resultados similares fueron reportados por Giordano (2016), cuando refiere que "el retroceso de las exportaciones globales y regionales resultó de reducciones en el valor de las importaciones tanto de los países desarrollados como de los países en desarrollo y de intensas presiones deflacionarias que afectaron los precios de las ventas externas".

Asimismo, las PYMES estudiadas de acuerdo a sus reportes permiten inferir que son tímidas las iniciativas en los procesos de aprendizaje organizacional para una performance exportadora, de acuerdo a los requerimientos de los mercados externos. No obstante, de acuerdo a Moori-Koenig et al (1994), de acuerdo a una evidencia empírica muestra que entre las primeras operaciones de comercio exterior y la consolidación de la estrategia exportadora como una actividad regular de las empresas transcurre un período de no menos de diez años. Según estos autores este comportamiento es de hecho un proceso paulatino que deber ser considerado como un esfuerzo de maduración de cierta inelasticidad respecto al tiempo por lo que los resultados no pueden medirse en un período corto y en especial cuando las performances externas cambian mucho.

En síntesis, los resultados obtenidos en este grupo de PYMES estudiadas permiten establecer que producen productos especializados y con condiciones técnicas de acuerdo a los mercados de exportación. No existe relación entre el tamaño y 
el grado de inserción esto último está de acuerdo con los reportes encontrados en la revisión de antecedentes. Asimismo, una ambigüedad exportadora dentro de los diez años o superior lo que les hace empresas con poca madurez en la dinámica de aprendizaje exportador.

En referencia a la variable conducta exportadora, los resultados permiten establecer que las Pymes están sometidas a desiguales condiciones de financiamiento que sus competidores, en especial muy influenciadas por el riesgo país, poca tecnología de punta disponible, muchas variaciones en la actualización de procesos, así como la disposición de materia prima de calidad, lo que trae como consecuencia un alto esfuerzo para adaptar los productos a las exigencias de los mercados externos. Entre las dimensiones estudiadas la tipología exportadora está en función a la búsqueda de la excelencia exportadora, tener claros planes de senderos de excelencia que a su vez permita un predominio en el mercado mejorando así las oportunidades, cuyos reportes son muy altos.

Por otra parte, elementos como la imaginación, creatividad y construcción son de los factores que reportan una buena presencia, dejando una brecha de mejoramiento de ya que podría tener una tendencia a actitud "pasiva" en la búsqueda de nuevos clientes. Al respecto, Salvador y Heijs (2005), asocian la conducta exportadora a altos niveles de innovación manifiesta por parte de las PYMES, rasgos que no fueron observados en la mayoría de la muestra objeto de estudio. Así también es importante considerar que dentro de la conducta exportadora, los conceptos de calidad, como lo afirma Cárdenas (2016) van más allá del cumplimiento de ciertas especificaciones, puesto que esto no asegura la satisfacción del cliente.

\section{Conclusiones}

En conclusión, los resultados obtenidos en este estudio muestran que fue posible caracterizar el perfil de datos básicos en las empresas objeto de estudio, siendo PYMES que producen productos especializados para la exportación, respondiendo a las especificaciones técnicas y estándares reglamentarios de los mercados de exportación. Asimismo, el tamaño y grado de inserción permite caracterizarlas como PYMES en desarrollo, lo que está en concordancia con su ambigüedad de exportación la cual se encuentra en un rango de 10 a 2 años frente a empresas creadas hace más de 20 años.

En referencia a poder identificar los 
indicadores claves de la performance existen debilidades en los procesos de aprendizaje organizacional para la exportación, así como no son constantes las iniciativas para fomentar la creatividad de nuevos productos, que puedan atender a nuevos clientes así no siempre están claras las exigencias de los mercados de destino.

Dentro de los procesos de planificación, los hallazgos permiten afirmar que los procesos de planificación de exportación, así como la logística de facilitación de medios de exportación, permisología y normativas de calidad son más impositivas que de búsqueda de un sendero sostenido de calidad.

Continuando con el analizar la tipología exportadora se concluye que son desiguales las condiciones de financiamiento en los mercados de destino, así como afecta las indexaciones de riesgo país sobre Venezuela. Dentro de los factores endógenos que sustentan el posicionamiento competitivo se encuentran, la aplicación de tecnologías, la actualización de procesos y la relación calidad precio, lo que incide en un esfuerzo para poder adaptar los productos al mercado externo, requiriendo de un alto grado de complejidad de las estrategias de exportación.

En cuanto a evaluar la actitud proactiva o pasiva se puede concluir que la falta de madurez y antigüedad exportadora, así como la inexistencia unidades de planeación estratégica para la exportación permiten afirmar que la actitud más llegada a "pasiva", ya que responde más a los procesos de desindustrialización de país, así como una estrategia de compensación de capitales que mantengan la actividad económica de las empresas, frente a las políticas de estado imperantes en el momento de desarrollo de este estudio.

Finalmente, en términos generales se puede concluir que fue posible la aplicación del modelo Moori- Koenig- Youguel para determinar el perfil de la inserción externa y conducta exportadora, siendo esta en términos generales que la inserción externa de las PYMES responde más a una estrategia particular que a una política de Estado, que involucre inversiones productivas de estas como país y bajo convenios y/o acuerdos comerciales de cooperación. Esta situación conlleva a que la conducta exportadora caracterizada por poco compromiso con los senderos de excelencia, poca disponibilidad de mano de obra calificada en los diferentes niveles organizacionales, así como una tenue visión estratégica frente a los mercados externos, alejando de iniciativas de innovación y desarrollos 
constantes de I+D+Capital Humano.

\section{Referencias bibliográficas}

Alderete, M. (2007). Nuevas tecnologías de la información y de la comunicación: factores explicativos de la conducta exportadora en Argentina. Economía y sociedad, 12(20), 2.

Bucardo, A. (2007). Canalización de recursos de migrantes a proyectos productivos: Resultados de un programa piloto FOMIN. Inter-American Development Bank.

Cárdenas, A. (2016). Calidad en la gestión universitaria ecuatoriana: Una búsqueda latente de eficiencia educativa. Multiciencias, 16 (2), 194-201.

Carrasquero, E. (2012). Herramienta para la toma de decisiones en organizaciones públicas. Ciencias de Gobierno, 5(1).

Coba, E.; Díaz, J. \& Sánchez, A. (2016). Factores de éxito en la gestión de las asociaciones. un estudio del programa hilando el desarro1lo. Revista de Investigación Sigma, 3(1).

Estrada, S. \& Heijs, J. (2003). Innovación tecnológica y competitividad: análisis microeconómico de la conducta exportadora en México.

Estrada, S. \& Heijs, J. (2005). Comportamiento innovador y competitividad: factores explicativos de la conducta exportadora en México. El caso de Guanajuato. Problemas del desarrollo, 36(143), 113-143.

Hurtado, R.; Charum, J.; Rivera, Á.: Daza, S.; Silva, A.; Llanos, E. \& Bernal, A. (2016).
Evaluación Programa Nacional de desarrollo científico y tecnológico BID Etapa III (1995-2003). (BID/CO-134-Contrato 085/0C-00). República de Colombia (1995).

Lora, E. (2013). La Realidad Social: Una introducción a los Problemas y Políticas del Desarrollo Social en América Latina: Módulo V: Se Buscan Buenos Empleos.

Niembro, A. (2017). Una tipología de empresas latinoamericanas exportadoras deservicios intensivos en conocimiento y los determinantes de su competitividad internacional. Estudios Gerenciales, 33(142), 64-75.

Moori, V.; Yoguel, G.; Milesi, D. \& Robert, V. (2007). Desarrollo de ventajas competitivas: pymes exportadoras exitosas en Argentina, Chile y Colombia. Revista de la CEPAL.

Moori, V.; Milesi, D. \& Yoguel, G. (2001). Ventajas competitivas dinámicas: las pymes exportadoras exitosas argentinas. Fundes Argentina, Buenos Aires.

Moori, V. \& Yoguel, G. (1995). Perfil de la inserción externa y conducta exportadora de las pequeñas y medianas empresas industriales argentinas.

Pelekais, C.; Finol, M.; Neuman, N.; Carrasquero, E., García, J., \& Leal, M. (2012). El $\mathrm{ABC}$ de la investigación. Un encuentro con la ciencia. Venezuela. Astro data, 308. 